\title{
Hyaluronidases and hyaluronan synthases expression is inversely correlated with malignancy in lung/bronchial pre-neoplastic and neoplastic lesions, affecting prognosis
}

\author{
V.K. de Sá ${ }^{1}$, T.P. Rocha ${ }^{1}$, A.L. Moreira ${ }^{2}$, F.A. Soares ${ }^{3}$, T. Takagaki $^{4}$, L. Carvalho $^{5}$, \\ A.G. Nicholson ${ }^{6}$ and V.L. Capelozzi ${ }^{1}$ \\ ${ }^{1}$ Departamento de Patologia, Faculdade de Medicina, Universidade de São Paulo, São Paulo, SP, Brasil \\ ${ }^{2}$ Memorial Sloan-Kettering Cancer Center, New York, NY, USA \\ ${ }^{3}$ Departamento de Anatomia Patológica, A.C. Camargo Cancer Center, São Paulo, SP, Brasil \\ ${ }^{4}$ Divisão de Pneumologia, Instituto do Coração, Faculdade de Medicina, Universidade de São Paulo, São Paulo, SP, Brasil \\ ${ }^{5}$ Universidade de Coimbra, Coimbra, Portugal \\ ${ }^{6}$ Royal Brompton and Harefield Hospitals, NHS Foundation Trust, National Heart and Lung Division, Imperial College, London, UK
}

\begin{abstract}
We collected a series of 136 lung/bronchial and 56 matched lung parenchyma tissue samples from patients who underwent lung/bronchial biopsies and presented invasive carcinoma after lung surgery. The lung/bronchial samples included basal cell hyperplasia, squamous metaplasia, moderate dysplasia, adenomatous hyperplasia, severe dysplasia, squamous cell carcinoma and adenocarcinoma. Matched lung parenchyma tissue samples included 25 squamous cell carcinomas and 31 adenocarcinomas. Immunohistochemistry was performed to analyze for the distribution of hyaluronidase (Hyal)-1 and -3 , and hyaluronan synthases (HAS)-1, -2 , and -3 . Hyal-1 showed significantly higher expression in basal cell hyperplasia than in moderate dysplasia $(P=0.01)$, atypical adenomatous hyperplasia $(P=0.0001)$, or severe dysplasia $(P=0.03)$. Lower expression of Hyal-3 was found in atypical adenomatous hyperplasia than in basal cell hyperplasia $(P=0.01)$ or moderate dysplasia $(P=0.02)$. HAS-2 was significantly higher in severe dysplasia $(P=0.002)$ and in squamous metaplasia $(P=0.04)$ compared with basal cell hyperplasia. HAS-3 was significantly expressed in basal cell hyperplasia compared with atypical adenomatous hyperplasia $(P=0.05)$ and severe dysplasia $(P=0.02)$. Lower expression of HAS-3 was found in severe dysplasia compared with squamous metaplasia $(P=0.01)$ and moderate dysplasia $(P=0.01)$. Epithelial Hyal -1 and -3 and HAS $-1,-2$, and -3 expressions were significantly higher in pre-neoplastic lesions than in neoplastic lesions. Comparative Cox multivariate analysis controlled by $\mathrm{N}$ stage and histologic tumor type showed that patients with high HAS-3 expression in pre-neoplastic cells obtained by lung/ bronchial biopsy presented a significantly higher risk of death $(\mathrm{HR}=1.19 ; \mathrm{P}=0.04)$. We concluded that localization of Hyal and HAS in lung/bronchial pre-neoplastic and neoplastic lesions was inversely related to malignancy, which implied that visualizing these factors could be a useful diagnostic procedure for suspected lung cancer. Finalizing this conclusion will require a wider study in a randomized and prospective trial.
\end{abstract}

Key words: Hyaluronidases and hyaluronan synthases; Lung cancer; Pre-neoplastic lung/bronchial lesions; Immunohistochemistry; Morphometry; Prognosis

\section{Introduction}

Lung cancer remains the leading cause of cancer death worldwide. At the time of diagnosis, lung cancer is usually extensive, and despite improvements in therapy, the overall 5-year survival rate for lung cancer patients remains less than $15 \%$ (1). The major reasons for the poor prognosis for lung cancer are the lack of effective screening and early diagnosis procedures, the propensity for early metastasis and the inability of systemic therapies to cure patients with widely metastatic disease (2).

Lung cancer is the result of a multi-step accumulation of genetic and/or epigenetic alterations. Better understanding of the molecular mechanisms by which these alterations affect lung cancer pathogenesis could provide new diagnostic procedures and prognostic factors for

Correspondence: V.L. Capelozzi: <vcapelozzi@lim05.fm.usp.br>. 
detection of early-stage or recurrent disease. In this regard, many have investigated molecular markers in pre-neoplastic and neoplastic lesions to gain insight into tumor recurrence and shortened survival (3). Because degradation of the extracellular matrix is important to invasion, cellular motility, and proliferation, several glycosaminoglycans have been targeted as potentially useful tumor markers. Among these, hyaluronidases (Hyal) and hyaluronan synthases (HAS) have shown to be promising markers. Increased hyaluronidase expression has also been reported in colon $(4,5)$, prostate (6), ovarian (7) endometrium (8), breast $(9,10)$, skin (11), and lung (12-14) cancers. HAS is a group of isozymes that synthesizes hyaluronan (HA), a glycosaminoglycan abundant in connective tissue and also present in several types of epithelia $(15,16)$ where it controls cell migration, differentiation and proliferation (17). Three types of HAS are known: HAS-1, HAS-2, and HAS-3 (18). Therefore, upregulated HAS expression is a likely contributor to $\mathrm{HA}$ accumulation in tissues, and promotes tumor growth (4) and metastasis, especially when co-expressed with Hyal (19). Different types of Hyal - Hyal-1, Hyal-2, and Hyal-3 - can degrade the long chains of alternating units of $\mathrm{N}$-acetylglucosamine and glucuronic acid into different-sized molecules. Hyal degrades HA into fragments of 20-25 disaccharides, which are needed to activate the MAP-kinase pathway (20). The major mRNA transcript of Hyal-3 is enzymatically inactive and appears to have only a supportive role in Hyal-1 expression (21).

Expression of epithelial HA, Hyal, and HAS is modulated in premalignant and malignant esophageal, breast, ovarian, and lung tumors (22-24). We hypothesized that Hyal and HAS expressions also change in bronchial metaplasia and dysplasia, and in atypical adenomatous hyperplasia, which are thought to be stages in the development of squamous cell lung carcinoma $(25,26)$ and adenocarcinoma, respectively. To test this hypothesis, we collected samples of metaplastic, dysplastic, and atypical adenomatous hyperplasia lesions and malignant lung tumors and stained them for Hyal and HAS.

\section{Material and Methods}

\section{Histologic specimens}

We retrospectively obtained 136 lung specimens of pre-neoplastic lesions from endoscopic biopsies and lung resections removed from 56 patients who were diagnosed with lung cancer and had undergone diagnostic and/or surgical treatment. Endoscopic biopsies or lung resections were performed under general anesthesia in the Hospital das Clínicas, AC Camargo Cancer Center, University of Coimbra, and Brompton Hospital in London, from 2008 to 2011. For endoscopic biopsies, a flexible bronchoscope (model 1Q10, Olympus Corp., Japan) was used. The bronchoscope was directly used at sites of nodules or masses. Typically, three or more samples were obtained. After this procedure, specimens were fixed in $10 \%$ buffered formalin for periods of $4-16 \mathrm{~h}$ and embedded in paraffin according to the routine of the laboratory involved. Staining by hematoxylin and eosin, and mucicarmine or PAS with diastase was performed on $5-\mu \mathrm{m}$ sections in all specimens embedded in paraffin and reviewed by two pathologists (VKS and VLC). Slides resulting from the processing of each specimen were obtained from the pathology files of each institution for review and revision. The 2015 World Health Organization lung tumor classification (27) was used to classify basal cell hyperplasia $(n=64)$, squamous metaplasia $(n=13)$, moderate dysplasia $(n=14)$, severe dysplasia $(n=9)$, and atypical adenomatous hyperplasia $(n=36)$. The invasive carcinomas consisted of 25 squamous cell carcinomas, and 31 adenocarcinomas. For evaluation of staining coverage and intensity, the tissue sections were stained for HAS-1, -2 , and -3 and Hyal-1, -2 , and -3 as described below. Table 1 summarizes the patient characteristics.

The project was approved by the Ethics Committee for Research Project Analysis (CAPPesq) from the Diretoria Clínica do Hospital das Clínicas and Faculdade de Medicina, Universidade de São Paulo, São Paulo, SP, Brazil.

Table 1. Patient characteristics.

\begin{tabular}{lc}
\hline Characteristics & $\mathrm{n}=56$ \\
\hline Median age, years (range) & $64(43-84)$ \\
Gender & \\
Male & 35 \\
Female & 21 \\
Median tobacco history, pack/years (range) & $37(0-100)$ \\
Overall stage & \\
I & 36 \\
II & 16 \\
III & 4 \\
N stage & \\
$\mathrm{N}_{0}$ & 42 \\
$\mathrm{~N}_{1}$ & 14 \\
Treatment & \\
Surgery & 56 \\
Surgery + chemotherapy & 4 \\
Histological types & \\
Squamous cell carcinoma & 25 \\
Adenocarcinoma & 31 \\
Median follow-up, months (range) & $16(3-30)$ \\
Patients censored for survival analysis at last & 18 \\
follow-up time & \\
\hline
\end{tabular}

Data are reported as number of the 56 patients out of 136 who underwent lung/bronchial biopsies and presented invasive carcinoma after lung surgery. 


\section{HAS and Hyal staining}

To avoid antigen decay, serial slide sections from formalin-fixed paraffin-embedded (FFPE) tissues were paraffin coated and stored at $4{ }^{\circ} \mathrm{C}$ during a median period of 4 years (i.e., from 2008 to 2011) in the different centers included in the study.

Immunohistochemistry was performed to detect Hyal-1, -3 and HAS-1-3 antigens in pre-neoplastic and neoplastic lesions, using monoclonal antibody Hyal-1 (1D10), Hyal-3 (E-11), HAS-1 (C-14), HAS-2 (S-15), and HAS-3 (E-15), all from Santa Cruz Biotechnology (USA). Briefly, silanized slides containing tissue sections of $3 \mu \mathrm{m}$ were used in all cases. The sections were deparaffinized in xylol, rehydrated in an alcohol gradient, and stored in $0.05 \mathrm{M}$ sodium phosphate buffer (PBS), pH 7.2-7.4. The sections were then subjected to antigen retrieval in a microwave oven. Next, the slides were incubated overnight with the respective antibodies in concentrations previously established (1:200), washed in 0.05 M PBS, $\mathrm{pH} 7.2-7.4$, and incubated with the secondary antibody, using a large streptavidin-avidin-biotin-peroxidase system (k-0690; Dako A/S, Denmark). Diaminobenzidine (Sigma Diagnostics, USA) was used as a chromogen, and the sections counterstained with hematoxylin. Intense brown cytoplasmic staining in pre-neoplastic and neoplastic lesions was considered positive for Hyal-1 and -3 and HAS-1-3.

\section{Evaluation of immunostaining}

After the immunohistochemical reaction, markers in tumors and pre-neoplastic lesions were quantified using the Automated Cellular Imaging System (ACIS) III instrument (Dako, USA). Briefly, ACIS III consists of an automated digital microscope and a computer with a 26-image capture and image processing system. Each immunohistochemically stained slide was scanned and the images were reviewed on the computer screen. The ACIS III can detect, count and classify cell types based on levels of hue, saturation and brightness. The signal is then converted to number density measurement. The computer software "membranes and cytoplasm", which is part of the system, was used to analyze protein expression by measuring the staining intensity of the cytoplasm and cell membranes and adjusting the threshold to the pixels showing immunoreactivity or not. The results are reported in continuous variables ranging from 0 to 256 . The areas to be analyzed on each slide were selected manually using the selection tools of the ACIS III. For statistical analyses, we used the average of two regions (stroma and tumor) of each case (24).

\section{Statistical analysis}

Statistical analysis was performed using PASW Statistics for Windows, Version 18.0 (SPSS Inc., USA). When necessary, variables were analyzed with the KolmogorovSmirnov test to determine the normality pattern. ANOVA tests were used to analyze Hyal-1 and -3 and HAS-1-3 immunoexpression in pre-neoplastic and neoplastic lesions. When non-parametric methods were used, simultaneous comparisons of confidence were corrected with Bonferroni's posttest. The Spearman test was used to clarify relationships between Hyal-1 and -3 and HAS-1, -2 , and -3 staining with pre-neoplastic variables studied. Receiver operation characteristic (ROC) curves were developed to determine optimal cut-off limits that yielded the best possible sensitivity and specificity values. Data on surgical and pathologic parameters, and Hyal and HAS staining inferences, were analyzed by the Cox proportional hazards model, using single-variable analysis (univariate analysis). Stratified Kaplan-Meier analyses were performed on the variables found to be significant in the multivariate Cox proportional hazards model. Results for which $\mathrm{P} \leqslant 0.05$ were considered to be significant.

\section{Results}

Cells showing Hyal-1- or Hyal-3-positive immunostaining were mainly epithelial cells, whereas most stromal cells showed negative or weak expression (Figure 1). Hyaluronidase-positive staining was localized intracellularly, spreading diffusely throughout the cytoplasm (Figure 2). Immunostaining with specific antibodies for HAS-1-3 showed positive staining in all samples, regardless of the lesion type (Figure 2). The HAS-1-3 proteins were detected homogenously in the cytoplasm and at the plasma membrane (Figure 2).

In all tissue groups, Hyal-1-positive epithelial cells ranged from $15.81 \pm 11.16$ to $82.56 \pm 11.96$ (Table 2). The percentage of Hyal-1 was significantly higher in basal cell hyperplasia compared with moderate dysplasia $(P=0.02)$, adenomatous hyperplasia $(P=0.0001)$, severe dysplasia $(P=0.05)$, squamous cell carcinoma $(P=0.0001)$, and adenocarcinoma $(P=0.0001$; Figure $3 A)$. Adenomatous hyperplasia expressed less Hyal-1 than did squamous metaplasia $(P=0.004)$, squamous cell carcinoma $(P<0.01)$ or adenocarcinoma $(P<0.01$; Figure $3 A)$. The percentage of Hyal-3-positive epithelial cells ranged from $47.90 \pm 22.41 \%$ to $79.49 \pm 23.51$ (Table 2). Hyal-3 staining intensity in epithelial cells was weak in atypical adenomatous hyperplasia, moderate in basal cell hyperplasia and strong in moderate hyperplasia (Figure 3B). The percentage of positive cells in the pre-neoplastic lesions and tumor cells also differed among the HAS isoforms (Table 2; Figures 2 and $3 \mathrm{C}$ ). The proportion of HAS-1-positive epithelial cells tended to be significantly lower in squamous cell carcinoma and adenocarcinomas than in pre-neoplastic lesions ( $P=0.05$; Figure $3 C$ ). HAS-1 percentage in basal cell hyperplasia was $63.14 \pm 20.18$ whereas average HAS-1-positive cell percentages in other pre-neoplastic lesions were squamous metaplasia: $64.84 \pm$ 16.60; moderate dysplasia: $66.32 \pm 13.96$; atypical adenomatous hyperplasia: $46.63 \pm 31.81$; and severe dysplasia: $51.61 \pm 22.54(\mathrm{P}<0.05)$. For squamous cell 


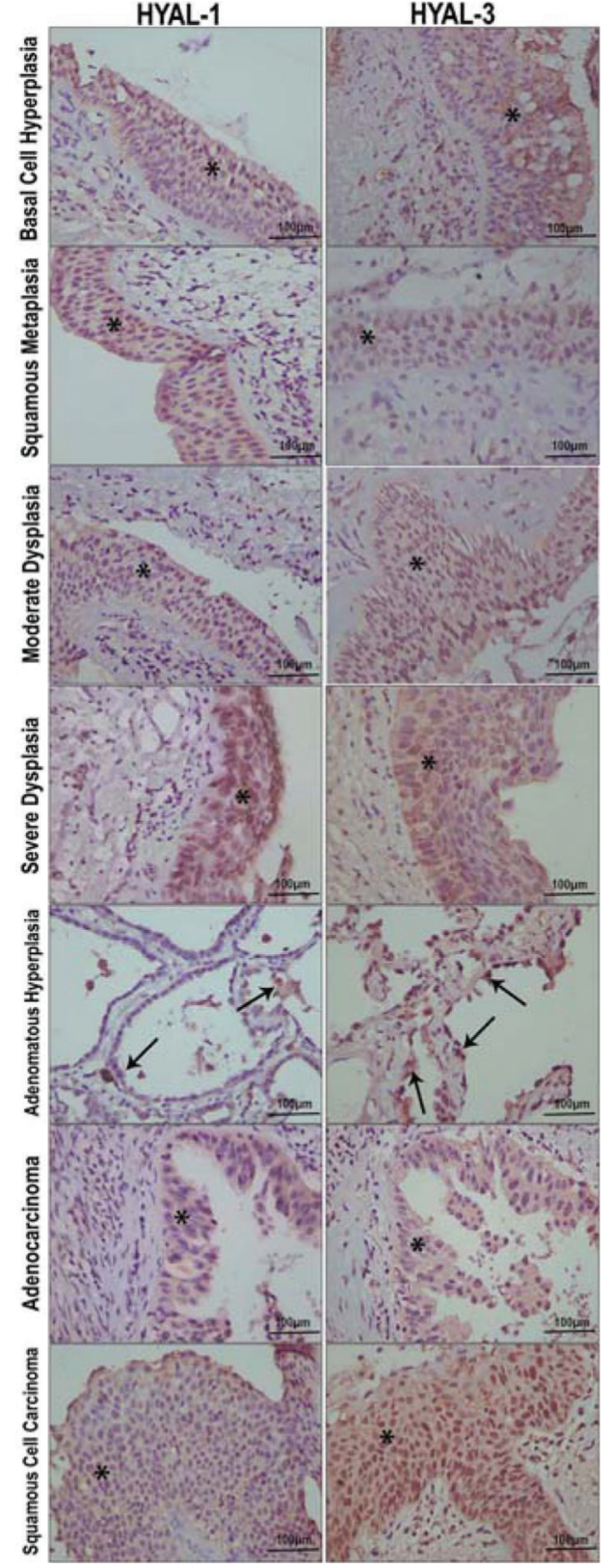

Figure 1. Hyaluronidase (Hyal)-1 and -3 in basal cell hyperplasia, squamous metaplasia, moderate dysplasia, atypical adenomatous hyperplasia, severe dysplasia, squamous cell carcinoma and adenocarcinoma, as shown by immunohistochemistry staining. Hyal-1 was more prominently expressed by epithelial cells in basal cell hyperplasia than in moderate dysplasia, adenomatous hyperplasia, severe dysplasia, squamous cell carcinoma or adenocarcinoma. Hyal-1 expression decreased and became more dispersed as malignancy increased and epithelial cells became less organized. Strong and diffuse expression of Hyal-3 was seen in atypical adenomatous hyperplasia, basal cell hyperplasia and moderate dysplasia. Arrows and asterisks indicate cytoplasmic expression in epithelial cells of pre-neoplastic and neoplastic tissue. Bar: $100 \mu \mathrm{m}$.

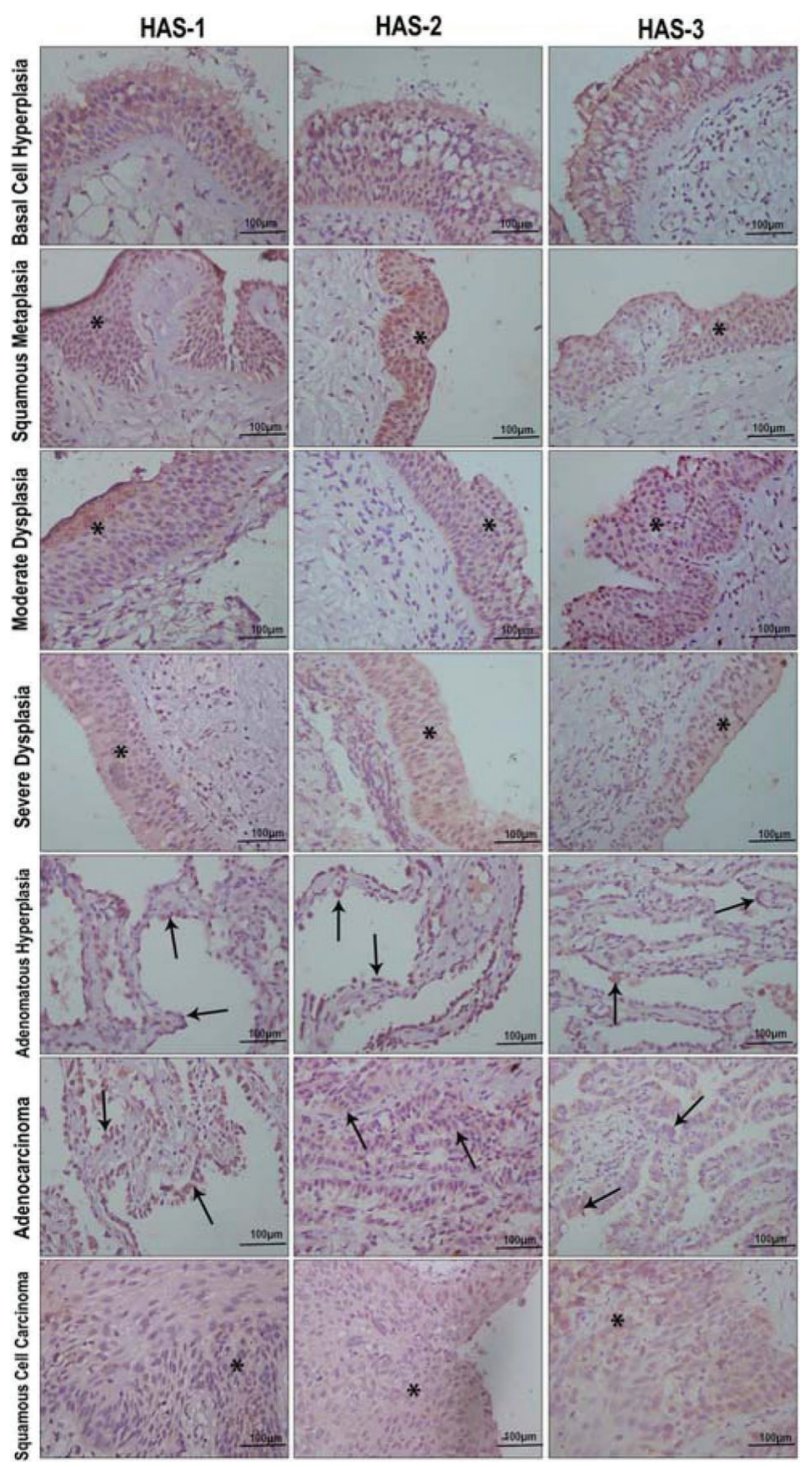

Figure 2. Hyaluronan synthases (HAS)-1, -2 , and -3 in basal cell hyperplasia, squamous metaplasia, moderate dysplasia, atypical adenomatous hyperplasia, severe dysplasia, squamous cell carcinoma and adenocarcinoma, shown by immunohistochemistry staining. Similar expression of HAS-1 was seen in basal cell hyperplasia, squamous metaplasia, moderate dysplasia, atypical adenomatous hyperplasia and severe dysplasia. Fewer epithelial cells in squamous cell carcinoma and adenocarcinoma expressed HAS-1. Numerous epithelial cells expressed HAS-2 in severe dysplasia compared to basal cell hyperplasia, squamous metaplasia and basal cell hyperplasia. HAS-3 was prominently expressed in basal cell hyperplasia, atypical adenomatous hyperplasia and severe dysplasia. Few epithelial cells expressed HAS-3 in severe dysplasia compared to squamous metaplasia and moderate dysplasia. Arrows and asterisks indicate cytoplasmic expression in epithelial cells of pre-neoplastic and neoplastic tissue. Bar: $100 \mu \mathrm{m}$. 
Table 2. Distribution and staining intensity of Hyal-1 and -3 and HAS-1, -2 and -3 in pre-neoplastic and neoplastic epithelium.

\begin{tabular}{ll}
\hline Protein/lesion & Means \pm SD \\
\hline Hyaluronidase 1 & \\
Basal cell hyperplasia & $82.56 \pm 11.96$ \\
Squamous metaplasia & $58.31 \pm 23.26$ \\
Moderate dysplasia & $36.09 \pm 27.30$ \\
Adenomatous hyperplasia & $15.81 \pm 11.16$ \\
Severe dysplasia & $43.01 \pm 27.04$ \\
Squamous cell carcinoma & $29.90 \pm 15.04$ \\
Adenocarcinoma & $35.43 \pm 16.34$ \\
Hyaluronidase 3 & \\
Basal cell hyperplasia & $67.88 \pm 23.41$ \\
Squamous metaplasia & $71.87 \pm 29.54$ \\
Moderate dysplasia & $79.49 \pm 23.51$ \\
Adenomatous hyperplasia & $47.90 \pm 22.41$ \\
Severe dysplasia & $68.00 \pm 27.46$ \\
Squamous cell carcinoma & $56.80 \pm 31.19$ \\
Adenocarcinoma & $56.47 \pm 27.01$ \\
Hyaluronan synthase 1 & \\
Basal cell hyperplasia & $63.14 \pm 20.81$ \\
Squamous metaplasia & $64.84 \pm 16.60$ \\
Moderate dysplasia & $66.32 \pm 13.96$ \\
Adenomatous hyperplasia & $46.63 \pm 31.81$ \\
Severe dysplasia & $51.61 \pm 22.54$ \\
Squamous cell carcinoma & $21.78 \pm 17.15$ \\
Adenocarcinoma & $28.31 \pm 21.33$ \\
Hyaluronan synthase 2 & \\
Basal cell hyperplasia & $73.08 \pm 19.57$ \\
Squamous metaplasia & $83.45 \pm 7.53$ \\
Moderate dysplasia & $75.73 \pm 18.25$ \\
Adenomatous hyperplasia & $66.27 \pm 28.32$ \\
Severe dysplasia & $91.13 \pm 8.60$ \\
Squamous cell carcinoma & $17.69 \pm 17.44$ \\
Adenocarcinoma & $19.87 \pm 18.17$ \\
Hyaluronan synthase 3 & \\
Basal cell hyperplasia & \\
Squamous metaplasia & \\
Moderate dysplasia & \\
Adenomatous hyperplasia & \\
Severe dysplasia & \\
Squamous cell carcinoma & \\
Adenocarcinoma & \\
\hline
\end{tabular}

Data are reported as means $\pm S D$. Determination of Hyal and HAS expression was based on immunohistochemistry. Hyal-1 expression was significantly greater in basal cell hyperplasia than moderate dysplasia $(P=0.02)$, adenomatous hyperplasia $(P=0.0001)$, severe dysplasia $(P=0.05)$, squamous cell carcinoma $(P=0.0001)$ or adenocarcinoma $(P=0.0001)$. Adenomatous hyperplasia expressed less Hyal-1 than did squamous metaplasia $(P=0.004)$, squamous cell carcinoma $(P<0.01)$ or adenocarcinoma $(P<0.01)$. Hyal-3 staining in epithelial cells significantly differed between atypical adenomatous hyperplasia and basal cell hyperplasia $(P=0.01)$ and moderate dysplasia $(P=0.02)$.
Squamous cell carcinoma and adenocarcinoma show lower proportions of HAS-1-positive cells than do pre-neoplastic lesions $(P=0.0001)$. HAS-2 expression was significantly higher in severe dysplasia $(P=0.002)$ and in squamous metaplasia $(P=0.01)$ than in basal cell hyperplasia. HAS-3 was significantly higher in basal cell hyperplasia than atypical adenomatous hyperplasia $(P<0.05)$ and severe dysplasia $(P<0.05)$. HAS-3 expression was lower in severe dysplasia than in squamous metaplasia $(P<0.01)$ or in moderate dysplasia $(P<0.01)$. The independent samples t-test was used for statistical analysis.

carcinoma and adenocarcinoma, the proportion of HAS-1positive cells was typically less than 20 ( $P=0.0001$; Figure $3 \mathrm{C}$ ). Staining of HAS-2 was significantly higher in severe dysplasia $(P=0.002)$ and squamous metaplasia $(P=0.01)$ than basal cell hyperplasia (Figure 3D). HAS-3 showed significantly greater expression in basal cell hyperplasia than in atypical adenomatous hyperplasia $(P<0.05)$ or severe dysplasia $(P<0.05$; Figure $3 E)$. Lower HAS-3 expression was found in severe dysplasia than in squamous metaplasia $(P<0.01)$ or moderate dysplasia $(P<0.01$; Figure 3E).

Table 3 shows correlation analyses for Hyal-1 and -3 , and HAS-1, -2 , and -3 expression and lesion types. A significant direct association was found between Hyal-1 and HAS-1 $(R=0.40 ; \quad P=0.0001), H A S-2 \quad(R=0.40$. $P=0.0001)$ and HAS-3 ( $R=0.50 ; P=0.0001)$. Pre-neoplastic and tumor lesions showed inverse associations between Hyal-1 ( $R=-0.70 ; P=0.0001)$, HAS-1 $(R=-0.30$; $P=0.03)$, HAS-3 ( $R=-0.45 ; P=0.0001)$.

Comparative Cox multivariate analyses by $\mathrm{N}$ stage and tumor histology showed a significant association between poor survival and high pre-neoplastic cell HAS-3 levels ( $H R=1.19 ; P=0.04$; Table 4). We ranked the cases by ROC curve into two groups with distinctly different average survival times as illustrated by regression plots in Figure 4. The group with $<27.01 \%$ HAS-3 had a median survival time of 72 months, whereas those with $>27.01 \%$ HAS-3 had a median survival time of just 52 months after surgery.

\section{Discussion}

For the present study, we hypothesized that in preneoplastic lesions, and squamous cell carcinoma and adenocarcinoma subtypes - i.e., tumors with different behaviors - Hyal and HAS should modulate different malignancy-induced pathways that affect lung cancer carcinogenesis. By IHC staining, we found that Hyal-1 and -3 , and HAS-1-3 were significantly overexpressed by epithelial cells in pre-neoplastic lesions compared with tumor epithelial cells. In fact, heterogeneous hyaluronidase expression has been shown in malignancies, and shows promise as an indicator of disease progression.

Antigen decay in archival formalin-fixed paraffinembedded (FFPE) tissue sections for immunohistochemistry 


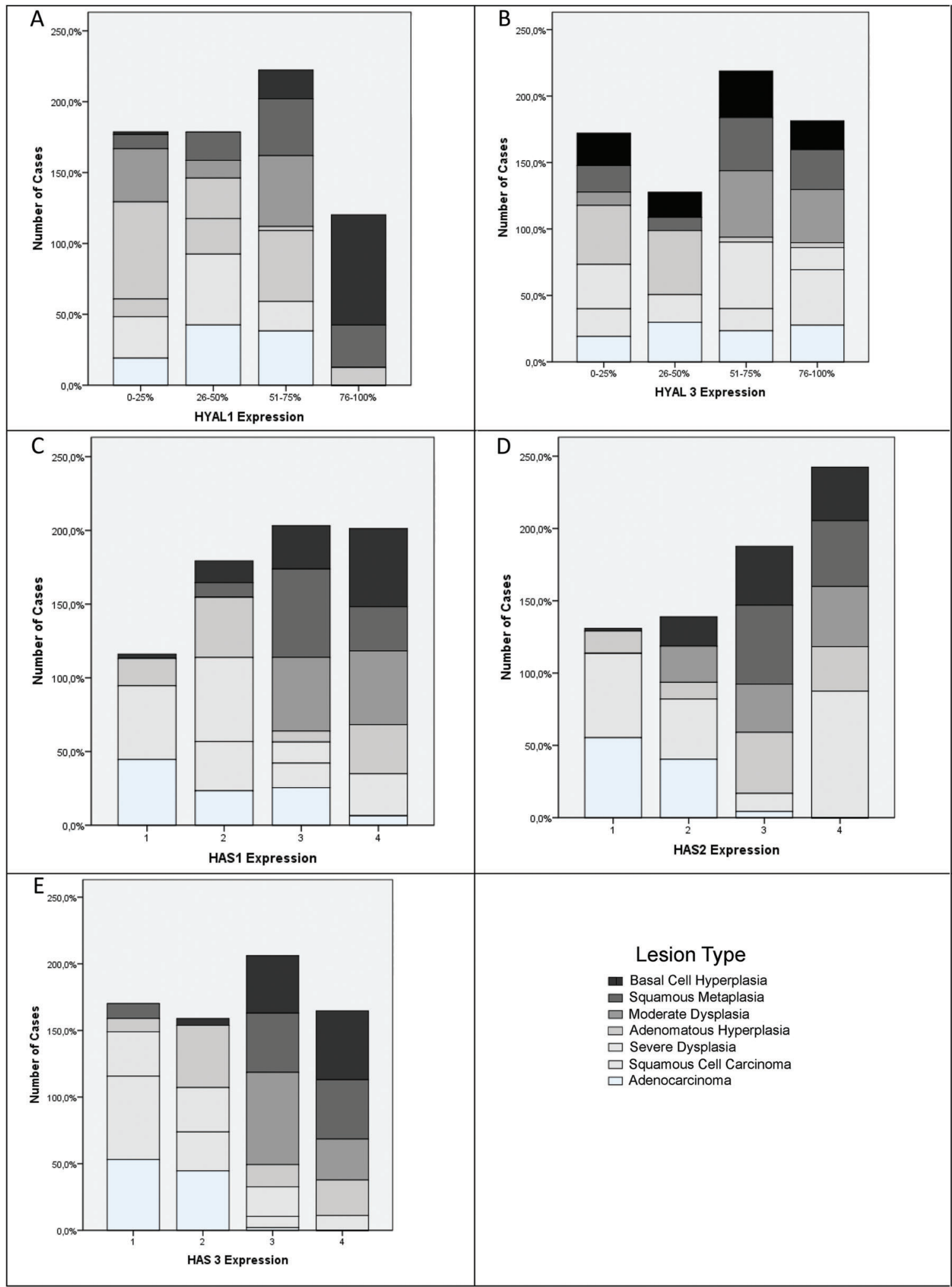

Figure 3. Intensity and coverage of Hyal-1 and -3 and HAS-1, -2 , and -3 staining in pre-neoplastic, squamous cell carcinoma and adenocarcinoma. See Results section for complete information about statistical comparisons (ANOVA). 
Table 3. Spearman correlation analysis of expression of Hyal-1 and -3 and HAS-1, -2 and -3 in pre-neoplastic and neoplastic lesion types.

\begin{tabular}{lcc}
\hline Neoplastic lesions & Pre-neoplastic lesions & Spearman correlation $(\mathrm{P})$ \\
\hline Hyal-1 & Hyal-1 & $\mathrm{R}=-0.70 ; \mathrm{P}=0.0001$ \\
HAS-1 & HAS-1 & $\mathrm{R}=-0.30 ; \mathrm{P}=0.03$ \\
HAS-3 & HAS-3 & $\mathrm{R}=-0.45 ; \mathrm{P}=0.0001$ \\
Hyal-1 & HAS-1 & $\mathrm{R}=0.40 ; \mathrm{P}=0.0001$ \\
Hyal-1 & HAS-2 & $\mathrm{R}=0.40 ; \mathrm{P}=0.0001$ \\
Hyal-1 & HAS-3 & $\mathrm{R}=0.50 ; \mathrm{P}=0.0001$ \\
\hline
\end{tabular}

stored at room temperature is a well-known phenomenon which may have affect translational and research studies; length of storage time appears central to this problem $(28,29)$. In the present study, this phenomenon had been previously minimized in the different centers where the samples were obtained for our study, as the serial slide sections from FFPE tissues were paraffin coated and cold stored at $4^{\circ} \mathrm{C}$ for a median time period of 4 years (i.e., from 2008 to 2011). Additionally, antigens that are nuclear or membranous (e.g., CD3, CD 31, CD117, estrogen and progesterone receptors, Ki67, p53, TTF-1, vimentin) show reduced immunosignals, whereas cytoplasmic antigens (smooth muscle actin, keratins 7, 20, AE1/AE3, 34ßE12, Hyal, and HAS) show little antigen decay.

We found that Hyal-1 expression was significantly increased in all pre-neoplastic lesions compared with malignant lesions. Similar results were previously reported by Siiskonen et al. (11), who found the proportion of Hyal1-positive melanocytic cells was significantly reduced in superficial and deep melanomas and also in lymph node metastases compared with in situ melanomas. In our specimens, the staining patterns of Hyal-1 differed between pre-neoplastic and malignant lesions, and the intensity of Hyal-1 in epithelial cells progressively decreased in malignant lesions compared with pre-neoplastic lesions. The presence of hyaluronidase in tumor cells has been shown to increase angiogenesis in vivo (12-14). Hyaluronan oligosaccharides produced by hyaluronidases mediate the angiogenic effects $(30,31)$ and may also activate matrix metalloproteinases, thus enhancing tumor invasiveness (32). Interestingly, in a mouse model of prostate cancer, co-expression of a Hyal-1 and a HAS-2 significantly increased angiogenesis (33). Upregulation of Hyal-1 and HAS-1, -2, and -3 in pre-neoplastic lesions was also observed in the present study.

The amount of hyaluronan seems to be biphasic in premalignant and malignant pulmonary lesions. First, in pre-neoplastic lesions, expression of HAS-1-3 in epithelial cells is greater; at this stage, hyaluronan levels vary among benign lesions, such as basal cell hyperplasia and squamous metaplasia. In dysplasia and atypical adenomatous hyperplasia, the proportion of HAS-2-positive epithelial cells is lower than in benign lesions, and at this state the hyaluronan content is also increased in cells of benign lesions. This may indicate accumulation of hyaluronan behind the intact basement membrane before the invasive phase has occurred. Instead, tumor cells show markedly reduced hyaluronan levels, which is associated with increased Hyal-1 expression. In fact, squamous cell carcinoma originates from the stratified epidermis as such. A similar tendency to increase hyaluronan staining in premalignant or early-stage malignant

Table 4. Cox proportional hazard model analysis of survival time (chi-square=20.17, $\mathrm{P}=0.005$ ).

\begin{tabular}{|c|c|c|c|c|c|c|c|}
\hline & \multirow[t]{2}{*}{ B } & \multirow[t]{2}{*}{ SE } & \multirow[t]{2}{*}{ Wald } & \multirow[t]{2}{*}{ Sig. } & \multirow[t]{2}{*}{$\operatorname{Exp}(B)$} & \multicolumn{2}{|c|}{$95 \% \mathrm{Cl}$ for $\operatorname{Exp}(\mathrm{B})$} \\
\hline & & & & & & Lower & Upper \\
\hline No Stage & -7.65 & 3.99 & 367 & 0.05 & 0.00 & 0.00 & 1.18 \\
\hline Squamous cell carcinoma & -1.21 & 1.46 & 0.68 & 0.40 & 0.29 & 0.01 & 5.25 \\
\hline \multicolumn{8}{|l|}{ Pre-neoplastic HYALs } \\
\hline HYAL1 & -0.00 & 0.08 & 0.01 & 0.91 & 0.99 & 0.83 & 1.17 \\
\hline HYAL3 & -0.07 & 0.04 & 2.64 & 0.10 & 0.92 & 0.85 & 1.01 \\
\hline \multicolumn{8}{|l|}{ Pre-neoplastic HAS } \\
\hline HAS1 & -0.05 & 0.04 & 1.50 & 0.22 & 0.94 & 0.87 & 1.03 \\
\hline HAS2 & -0.03 & 0.04 & 0.51 & 0.47 & 0.96 & 0.88 & 1.05 \\
\hline HAS3 & 0.17 & 0.10 & 3.02 & 0.04 & 1.19 & 0.97 & 1.44 \\
\hline
\end{tabular}

B: coefficient; SE: standard error; Sig.: significance; Exp(B): risk of B coefficient; Cl: confidence interval. 


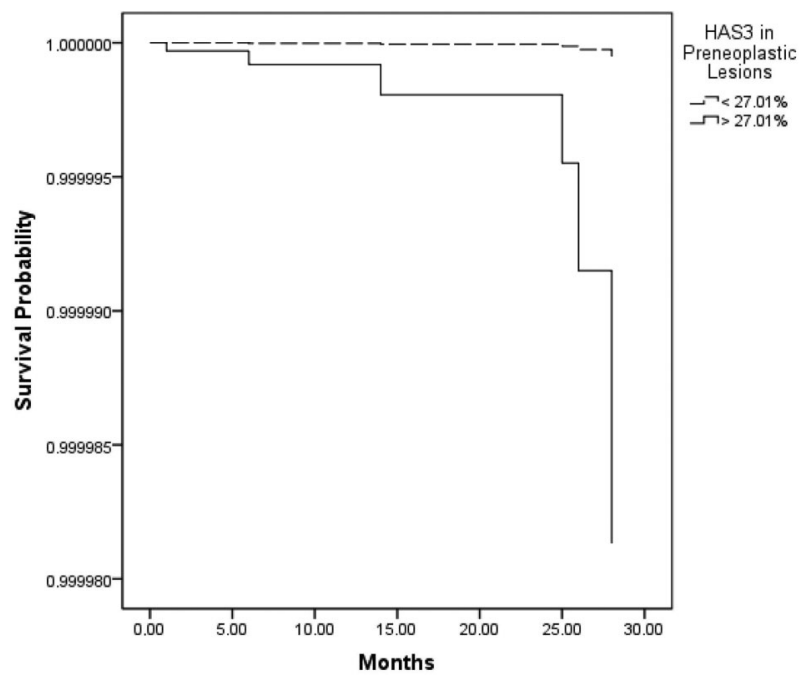

Figure 4. Regression plots of survival probability versus follow-up time in months for all patients. The dashed line indicates the group with $<27 \%$ HAS-3; the solid line indicates the group with $>27 \%$ HAS- 3 .

lesions and decreased staining in more advanced tumors has been reported earlier in squamous cell carcinomas of oral (34), laryngeal (35), esophageal (23), and skin (36) epithelium, all originating from stratified epithelium. Although adenocarcinoma originates from alveolar epithelial cells and not from stratified epithelium as such, a similar tendency to

\section{References}

1. Jemal A, Bray F, Center MM, Ferlay J, Ward E, Forman D. Global cancer statistics. CA Cancer J Clin 2011; 61: 69-90, doi: 10.3322/caac.v61:2.

2. Siegel R, Ma J, Zou Z, Jemal A. Cancer statistics, 2014. CA Cancer J Clin 2014; 64: 9-29, doi: 10.3322/caac.21208.

3. Herbst RS, Heymach JV, Lippman SM. Lung cancer. N Engl J Med 2008; 359: 1367-1380.

4. Jacobson A, Rahmanian M, Rubin K, Heldin P. Expression of hyaluronan synthase 2 or hyaluronidase 1 differentially affect the growth rate of transplantable colon carcinoma cell tumors. Int J Cancer 2002; 102: 212-219.

5. Simpson MA. Concurrent expression of hyaluronan biosynthetic and processing enzymes promotes growth and vascularization of prostate tumors in mice. Am J Pathol 2006; 169: 247-257.

6. Lokeshwar VB, Cerwinka WH, Isoyama T, Lokeshwar BL. HYAL1 hyaluronidase in prostate cancer: a tumor promoter and suppressor. Cancer Res 2005; 65: 7782-7789, doi: 10.1158/0008-5472.CAN-04-2805.

7. Nykopp TK, Rilla K, Sironen R, Tammi MI, Tammi RH, Hamalainen $\mathrm{K}$, et al. Expression of hyaluronan synthases (HAS1-3) and hyaluronidases (HYAL1-2) in serous ovarian carcinomas: inverse correlation between HYAL1 and hyaluronan content. BMC Cancer 2009; 9: 143, doi: 10.1186/14712407-9-143. increased hyaluronan staining in premalignant or early-stage malignant lesions and decreased staining in more advanced tumors has been reported in adenocarcinomas of oral (34), laryngeal (35), esophageal (22), and skin (11) epithelium, all originating from stratified epithelium.

Our data show, for the first time, the biphasic pattern of hyaluronan metabolism in lung tumors, and reveals increased hyaluronan synthesis in premalignant lesions followed by reduced hyaluronan content in squamous cell carcinoma and adenocarcinoma as a consequence of decreased HAS expression and increased degradative Hyal-1 and -3 . Further studies are needed to clarify the prognostic power of Hyal upregulation and HAS downregulation in lung tumors.

\section{Acknowledgments}

We are grateful to biologist Sandra de Morais Fernezlian, Laboratory of Immunohistochemistry for her technical assistance. Research supported by the National Council for Scientific and Technological Development (CNPq-471939/2010-2 and 483005/2012-6); Foundation for the Support of Research of the State of São Paulo (FAPESP 2011/52095-0); and the Laboratories for Medical Research, Hospital das Clinicas, University of São Paulo Medical School. AG Nicholson was supported by the National Institute of Health Research Respiratory Disease Biomedical Research Unit at the Royal Brompton and Harefield NHS Foundation Trust and Imperial College, London.

8. Nykopp TK, Rilla K, Tammi MI, Tammi RH, Sironen R, Hamalainen K, et al. Hyaluronan synthases (HAS1-3) and hyaluronidases (HYAL1-2) in the accumulation of hyaluronan in endometrioid endometrial carcinoma. BMC Cancer 2010; 10: 512, doi: 10.1186/1471-2407-10-512.

9. Bertrand P, Girard N, Duval C, d'Anjou J, Chauzy C, Menard $\mathrm{JF}$, et al. Increased hyaluronidase levels in breast tumor metastases. Int J Cancer 1997; 73: 327-331.

10. Udabage L, Brownlee GR, Nilsson SK, Brown TJ. The overexpression of HAS2, Hyal-2 and CD44 is implicated in the invasiveness of breast cancer. Exp Cell Res 2005; 310: 205-217, doi: 10.1016/j.yexcr.2005.07.026.

11. Siiskonen H, Poukka M, Tyynela-Korhonen K, Sironen R, Pasonen-Seppanen S. Inverse expression of hyaluronidase 2 and hyaluronan synthases $1-3$ is associated with reduced hyaluronan content in malignant cutaneous melanoma. BMC Cancer 2013; 13: 181, doi: 10.1186/1471-2407-13181.

12. de Sa V, Olivieri E, Parra ER, Ab'Saber AM, Takagaki T, Soares FA, et al. Hyaluronidase splice variants are associated with histology and outcome in adenocarcinoma and squamous cell carcinoma of the lung. Hum Pathol 2012; 43: 675-683, doi: 10.1016/j.humpath.2011.06.010.

13. de Sa V, Carvalho L, Gomes A, Alarcao A, Silva MR, Couceiro $\mathrm{P}$, et al. Role of the extracellular matrix in variations 
of invasive pathways in lung cancers. Braz J Med Biol Res 2013; 46: 21-31, doi: 10.1590/1414-431X20122263.

14. Rangel MP, de Sá VK, Martins V, Martins JR, Parra ER, Mendes A, Andrade PC, Reis RM, Longatto-Filho A, Oliveira CZ, Takagaki T, Carraro DM, Nader HB, Capelozzi VL. Tissue hyaluronan expression, as reflected in the sputum of lung cancer patients, is an indicator of malignancy. Braz J Med Biol Res 2015; 48: 557-567, doi: 10.1590/1414431X20144300.

15. Tammi R, Ripellino JA, Margolis RU, Tammi M. Localization of epidermal hyaluronic acid using the hyaluronate binding region of cartilage proteoglycan as a specific probe. $J$ Invest Dermatol 1988; 90: 412-414.

16. Tammi R, Tammi M, Hakkinen L, Larjava H. Histochemical localization of hyaluronate in human oral epithelium using a specific hyaluronate-binding probe. Arch Oral Biol 1990; 35: 219-224, doi: 10.1016/0003-9969(90)90058-I.

17. Toole BP. Glycosaminoglycans in morphogenesis. New York: Plenum; 1981.

18. Nedvetzki S, Gonen E, Assayag N, Reich R, Williams RO, Thurmond RL, et al. RHAMM, a receptor for hyaluronanmediated motility, compensates for CD44 in inflamed CD44knockout mice: a different interpretation of redundancy. Proc Natl Acad Sci U S A 2004; 101: 18081-18086, doi: 10.1073/ pnas. 0407378102.

19. Golshani R, Lopez L, Estrella V, Kramer M, lida N, Lokeshwar VB. Hyaluronic acid synthase-1 expression regulates bladder cancer growth, invasion, and angiogenesis through CD44. Cancer Res 2008; 68: 483-491, doi: 10.1158/0008-5472.CAN-07-2140.

20. Bharadwaj AG, Kovar JL, Loughman E, Elowsky C, Oakley GG, Simpson MA. Spontaneous metastasis of prostate cancer is promoted by excess hyaluronan synthesis and processing. Am J Pathol 2009; 174: 1027-1036.

21. Turley EA, Noble PW, Bourguignon LY. Signaling properties of hyaluronan receptors. J Biol Chem 2002; 277: 4589-4592, doi: 10.1074/jbc.R100038200.

22. Auvinen PK, Parkkinen JJ, Johansson RT, Agren UM, Tammi RH, Eskelinen MJ, et al. Expression of hyaluronan in benign and malignant breast lesions. Int J Cancer 1997; 74: 477-481.

23. Wang C, Tammi M, Guo H, Tammi R. Hyaluronan distribution in the normal epithelium of esophagus, stomach, and colon and their cancers. Am J Pathol 1996; 148: 1861-1869.

24. Pirinen RT, Tammi RH, Tammi MI, Paakko PK, Parkkinen JJ, Agren UM, et al. Expression of hyaluronan in normal and dysplastic bronchial epithelium and in squamous cell carcinoma of the lung. Int J Cancer 1998; 79: 251-255.
25. Pendleton N, Dixon GR, Green JA, Myskow MW. Expression of markers of differentiation in normal bronchial epithelium and bronchial dysplasia. J Pathol 1996; 178: 146-150.

26. Wang C, Tammi M, Tammi R. Distribution of hyaluronan and its CD44 receptor in the epithelia of human skin appendages. Histochemistry 1992; 98: 105-112, doi: 10.1007/BF00717001.

27. Travis WD, Brambilla E, Burke AP, Marx A, Nicholson AG. WHO classification of Tumours of the Lung, Pleura, Thymus and Heart. Lyon: 2015.

28. Grillo F, Pigozzi S, Ceriolo P, Calamaro P, Fiocca R, Mastracci L. Factors affecting immunoreactivity in long-term storage of formalin-fixed paraffin-embedded tissue sections. Histochem Cell Biol 2015 Jul;144(1):93-9, doi: 10.1007/ s00418-015-1316-4.

29. Nuovo AJ, Garofalo M, Mikhail A, Nicol AF, Vianna-Andrade C, Nuovo GJ. The effect of aging of formalin-fixed paraffinembedded tissues on the in situ hybridization and immunohistochemistry signals in cervical lesions. Diagn Mol Pathol 2013; 22: 164-173, doi: 10.1097/PDM.0b013e3182823701.

30. Liu D, Pearlman E, Diaconu E, Guo K, Mori H, Haqqi T, et al. Expression of hyaluronidase by tumor cells induces angiogenesis in vivo. Proc Natl Acad Sci U S A 1996; 93: 7832-7837, doi: 10.1073/pnas.93.15.7832.

31. Cui X, Xu H, Zhou S, Zhao T, Liu A, Guo X, et al. Evaluation of angiogenic activities of hyaluronan oligosaccharides of defined minimum size. Life Sci 2009; 85: 573-577, doi: 10.1016/j.lfs.2009.08.010.

32. Gao F, Liu Y, He Y, Yang C, Wang Y, Shi X, et al. Hyaluronan oligosaccharides promote excisional wound healing through enhanced angiogenesis. Matrix Biol 2010; 29: 107-116, doi: 10.1016/j.matbio.2009.11.002.

33. Fieber C, Baumann P, Vallon R, Termeer C, Simon JC, Hofmann $M$, et al. Hyaluronan-oligosaccharide-induced transcription of metalloproteases. J Cell Sci 2004; 117: 359-367, doi: 10.1242/jcs.00831.

34. Kosunen A, Ropponen K, Kellokoski J, Pukkila M, Virtaniemi $\mathrm{J}$, Valtonen $\mathrm{H}$, et al. Reduced expression of hyaluronan is a strong indicator of poor survival in oral squamous cell carcinoma. Oral Oncol 2004; 40: 257-263, doi: 10.1016/ j.oraloncology.2003.08.004.

35. Hirvikoski P, Tammi R, Kumpulainen E, Virtaniemi J, Parkkinen $\mathrm{JJ}$, Tammi M, et al. Irregular expression of hyaluronan and its CD44 receptor is associated with metastatic phenotype in laryngeal squamous cell carcinoma. Virchows Arch 1999; 434: 37-44, doi: 10.1007/s004280050302.

36. Karvinen S, Kosma VM, Tammi MI, Tammi R. Hyaluronan, CD44 and versican in epidermal keratinocyte tumours. Br J Dermatol 2003; 148: 86-94. 